\title{
Gestationsdiabetes (GDM) (Update 2019)
}

\author{
Alexandra Kautzky-Willer · Jürgen Harreiter · Yvonne Winhofer-Stöckl · Dagmar Bancher-Todesca • \\ Angelika Berger · Andreas Repa - Monika Lechleitner - Raimund Weitgasser
}

Zusammenfassung Gestationsdiabetes (GDM) wird als Glukosetoleranzstörung definiert, die erstmals in der Schwangerschaft entdeckt wird. GDM ist mit einer erhöhten fetomaternalen Morbidität sowie Langzeitkomplikationen bei Mutter und Kind assoziiert. Frauen, die die Kriterien eines manifesten Diabetes bereits in der Frühschwangerschaft erfüllen (Nüchternplasmaglukose $>126 \mathrm{mg} / \mathrm{dl}$, Spontanglukosemessung über $200 \mathrm{mg} / \mathrm{dl}$ oder $\mathrm{HbA}_{\mathrm{lc}}>6,5 \%$ vor der 20. Schwangerschaftswoche), sollen als Schwangere mit manifestem Diabetes klassifiziert und ebenso behandelt werden. Ein Screening auf unerkannten Typ-2-Diabetes bei der ersten pränatalen Kontrolle wird besonders bei Frauen mit hohem Risiko (Anamnese eines GDM oder Prädiabetes; Fehlbildungen, Totgeburt, wiederholte Aborte oder Geburtsgewicht über

\footnotetext{
A. Kautzky-Willer $(\bowtie) \cdot$ J. Harreiter $\cdot$ Y. Winhofer-Stöckl Gender Medicine Unit, Klinische Abteilung für Endokrinologie und Stoffwechsel, Universitätsklinik für Innere Medizin III, Medizinische Universität Wien, Währinger Gürtel 18-20, 1090 Wien, Österreich alexandra.kautzky-willer@meduniwien.ac.at

D. Bancher-Todesca

Klinische Abteilung für Geburtshilfe und feto-maternale Medizin, Universitätsklinik für Frauenheilkunde, Medizinische Universität Wien, Wien, Österreich

\section{A. Berger · A. Repa}

Klinische Abteilung für Neonatologie, Pädiatrische Intensivmedizin und Neuropädiatrie, Universitätsklinik für Kinder- und Jugendheilkunde, Medizinische Universität Wien, Wien, Österreich

\section{Lechleitner}

Interne Abteilung, Landeskrankenhaus Hochzirl - Natters,
} Hochzirl, Österreich

\section{R. Weitgasser}

Abteilung für Innere Medizin, Privatklinik

Wehrle-Diakonissen, Salzburg, Österreich
$4500 \mathrm{~g}$ in früheren Schwangerschaften; Adipositas, metabolisches Syndrom, Alter über 35 Jahre, bei Gefäßerkrankungen, Auftreten von Diabetessymptomen wie Glukosurie, ethnische Zugehörigkeit zu Gruppen mit hohem Risiko [arabisch, S- und SO-Asien, Lateinamerika]) empfohlen (Evidenzklasse B). GDM wird durch einen oralen Glukosetoleranztest (OGTT) oder durch Nüchternplasmaglukosekonzentrationen über $92 \mathrm{mg} / \mathrm{dl}$ diagnostiziert. Bei hohem Risiko kann ein OGTT (120 min; $75 \mathrm{~g}$ Glukose) bereits im ersten Trimenon sinnvoll sein, ist aber in jedem Fall bei allen Schwangeren mit bis dahin unauffälligen Glukosewerten zwischen der 24. und 28. Schwangerschaftswoche vorgeschrieben (Evidenzklasse B). Auf Basis der „Hyperglycemia and Adverse Pregnancy Outcome (HAPO) study" und nach den aktuellen WHO-Empfehlungen liegt ein GDM vor, wenn der Nüchternplasmaglukosewert $92 \mathrm{mg} / \mathrm{dl}$, der 1 -Stunden-Wert $180 \mathrm{mg} / \mathrm{dl}$ oder der 2-Stunden-Wert $153 \mathrm{mg} / \mathrm{dl}$ überschreiten (OGTT; Internationale Konsensuskriterien). Ein einziger erhöhter Wert ist für die Diagnose ausreichend und bedarf bereits einer strikten Stoffwechselkontrolle. Nach bariatrischer Operation wird aufgrund der Gefahr einer postprandialen Hypoglykämie die Durchführung eines OGTT nicht empfohlen. Alle Frauen mit GDM erhalten eine Ernährungsberatung und müssen ihre Blutzuckerwerte regelmäßig kontrollieren. Ebenso sollte, falls nicht kontraindiziert, die körperliche Aktivität erhöht werden. Falls die Blutzuckerspiegel nicht im Therapiebereich liegen (nüchtern $<95 \mathrm{mg} / \mathrm{dl}$ und $1 \mathrm{~h}$ nach den Mahlzeiten $<140 \mathrm{mg} / \mathrm{dl}$ ) soll als erste Wahl eine Insulintherapie initiiert werden. Neben der mütterlichen Stoffwechselüberwachung ist auch ein fetales Monitoring notwendig, um die mütterliche und fetale/neonatale Morbidität und die perinatale Mortalität möglichst gering zu halten. Alle Frauen mit GDM müssen 4 bis 12 Wochen nach der Entbindung neuerlich einen OGTT (75g; WHO-Kriterien) 
durchführen lassen, um ihre Glukosetoleranz neu zu klassifizieren. Bei Normalbefund soll der OGTT alle 2 Jahre wiederholt werden (Evidenzklasse B). Alle Frauen müssen über ihr (7-fach erhöhtes relatives) Risiko informiert werden, im weiteren Verlauf einen Typ-2-Diabetes zu entwickeln, sowie über mögliche Präventionsmaßnahmen. Dazu gehören Gewichtsreduktion bei Übergewicht, gesunde Ernährung und ausreichend körperliche Aktivität. Auch die Kinder sollen hinsichtlich einer unauffälligen Entwicklung regelmäßig nachuntersucht werden, und die ganze Familie soll über Lebensstilmaßnahmen zur Aufrechterhaltung/Verbesserung der Gesundheit informiert werden. Die regelmäßige Durchführung von geburtshilflichen Kontrollen sowie Ultraschalluntersuchungen wird empfohlen. Im Rahmen der neonatalen Untersuchungen müssen bei Neugeborenen von GDM-Müttern Blutzuckerkontrollen erfolgen und bei Erfordernis geeignete Maßnahmen eingeleitet werden.

Schlüsselwörter Gestationsdiabetes · Diabetische Fetopathie - Typ-2-Diabetes mellitus - Schwangerschaft · Schwangerschaftskomplikationen

\section{Gestational diabetes mellitus (Update 2019)}

Summary Gestational diabetes mellitus (GDM) is defined as a glucose tolerance disorder with onset during pregnancy and is associated with increased feto-maternal morbidity as well as long-term complications in mother and child. Women who fulfil the criteria of a manifest diabetes in early pregnancy (fasting plasma glucose $>126 \mathrm{mg} / \mathrm{dl}$, spontaneous glucose level $>200 \mathrm{mg} / \mathrm{dl}$ or $\mathrm{HbAlc}>6.5 \%$ before 20 weeks of gestation) should be classified as having manifest diabetes in pregnancy and treated as such. Screening for undiagnosed type 2 diabetes at the first prenatal visit (evidence level B) is particularly recommended in women at increased risk (history of GDM or prediabetes, malformation, stillbirth, successive abortions or birth weight $>4500 \mathrm{~g}$ in previous pregnancies, obesity, metabolic syndrome, age $>35$ years, vascular disease, clinical symptoms of diabetes, e.g. glucosuria, or ethnic groups with increased risk for GDM/T2DM, e.g. Arabian countries, south and southeast Asia and Latin America). A GDM is diagnosed by an oral glucose tolerance test (OGTT) or a fasting glucose concentration $\geq 92 \mathrm{mg} / \mathrm{dl}$. Performance of the OGTT $(120 \mathrm{~min}$, $75 \mathrm{~g}$ glucose) may already be indicated in the first trimester in high risk women but is mandatory between 24-28 gestational weeks in all pregnant women with previous non-pathological glucose metabolism (evidence level B). Based on the results of the Hyperglycemia and Adverse Pregnancy Outcome (HAPO) study and following the recent WHO recommendations, GDM is present if the fasting plasma glucose level exceeds $92 \mathrm{mg} / \mathrm{dl}$, the $1 \mathrm{~h}$ level exceeds $180 \mathrm{mg} / \mathrm{dl}$ or the $2 \mathrm{~h}$ level exceeds $153 \mathrm{mg} / \mathrm{dl}$ after glucose load- ing (OGTT international consensus criteria). A single increased value is sufficient for the diagnosis and a strict metabolic control is mandatory. After bariatric surgery an OGTT is not recommended due to the risk of postprandial hypoglycemia. All women with GDM should receive nutritional counselling, be instructed in self-monitoring of blood glucose and to increase physical activity to moderate intensity levels, if not contraindicated. If blood glucose levels cannot be maintained in the therapeutic range (fasting $<95 \mathrm{mg} / \mathrm{dl}$ and $1 \mathrm{~h}$ postprandial $<140 \mathrm{mg} / \mathrm{dl}$ ) insulin therapy should be initiated as first choice. Maternal and fetal monitoring is required in order to minimize maternal and fetal/neonatal morbidity and perinatal mortality. After delivery all women with GDM have to be re-evaluated by a $75 \mathrm{~g}$ OGTT (WHO criteria) 4-12 weeks postpartum to reclassify the glucose tolerance and every 2 years in cases of normal glucose tolerance (evidence level B). All women have to be informed about their (sevenfold increased relative) risk of developing type 2 diabetes (T2DM) at follow-up and possible preventive measures, in particular weight management, healthy diet and maintenance/increase of physical activity. Monitoring of the development of children and recommendations for a healthy lifestyle are necessary for the whole family. Regular obstetric examinations including ultrasound examinations are recommended. Within the framework of neonatal care, neonates of GDM mothers should undergo blood glucose measurements and if necessary appropriate measures should be initiated.

Keywords Gestational diabetes mellitus - Diabetic fetopathy · Type 2 diabetes mellitus · Pregnancy · Pregnancy complications

\section{Grundsatzstatement}

Frauen mit in der Schwangerschaft erstmals aufgetretener oder diagnostizierter Glukosetoleranzstörung haben gegenüber Schwangeren mit normaler Glukosetoleranz ein höheres Risiko an perinataler Morbidität und Mortalität, eine höhere Rate an operativen Entbindungen und ein höheres Risiko, postpartal einen Diabetes mellitus Typ 2 (T2DM) zu entwickeln [1, 2]. Frauen mit Gestationsdiabetes (GDM) und strikter metabolischer Kontrolle haben bessere Schwangerschaftsergebnisse als Frauen mit GDM, die nicht behandelt werden $[3,4]$. Patientinnen, bei denen sich während der Schwangerschaft ein Typ-1-Diabetes mellitus manifestiert, sowie solche mit einem bereits präkonzeptionell oder zu Schwangerschaftsbeginn bestehenden, aber erst in der Gravidität diagnostiziertem anderen Diabetestyp sollen wie Patientinnen mit präkonzeptionell bekanntem Diabetes mellitus behandelt und überwacht werden (s. Leitlinie: Gravidität bei vorbestehendem Diabetes). Ein präkonzeptionell bestehender T2DM kann angenommen werden, wenn bereits vor der 20. Schwangerschaftswoche 


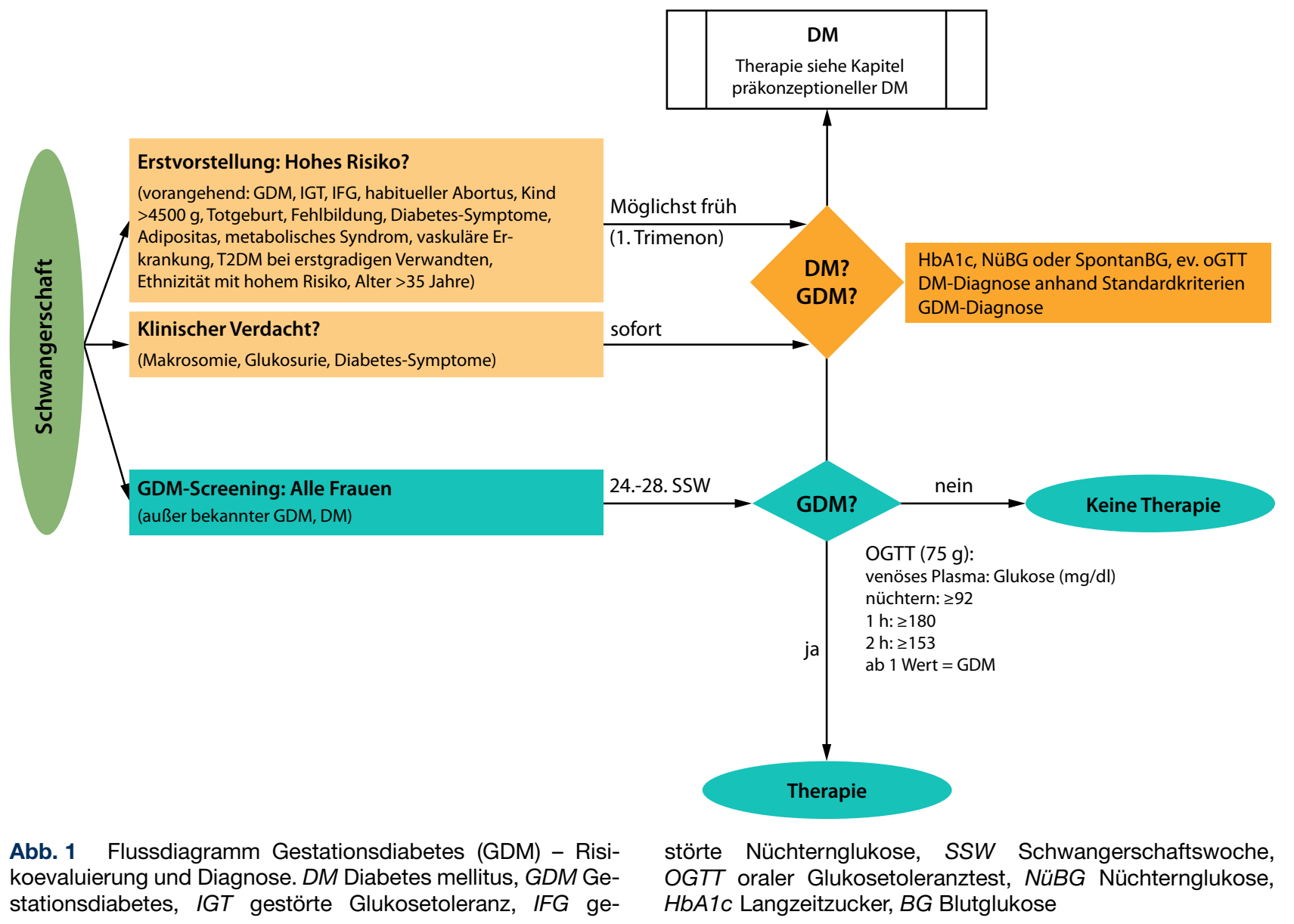

die Kriterien für einen manifesten Diabetes erfüllt werden (Nüchternblutzuckerwert $\geq 126 \mathrm{mg} / \mathrm{dl}$ oder Spontanmessungen über $200 \mathrm{mg} / \mathrm{dl}$; bzw. 2-h-Wert im oralen Glukosetoleranztest [OGTT] $\geq 200 \mathrm{mg} / \mathrm{dl}$ ) oder ein $\mathrm{HbA}_{1 \mathrm{c}}$ ab 6,5 \% gemessen wird. Frauen mit GDM haben postpartal ein deutlich erhöhtes Risiko für die Entwicklung eines T2DM und sollen deshalb über Präventionsmaßnahmen informiert und lebenslang überwacht werden.

\section{Risikoevaluierung und Diagnose}

Bei Erstvorstellung beim Frauenarzt wird die Schwangere bezüglich ihres Risikos für GDM oder Diabetes mellitus eingestuft. Bei Vorliegen eines höheren Risikos soll die Frau möglichst früh hinsichtlich einer Glukosestoffwechselstörung untersucht werden: Dies kann durch eine Nüchternglukosemessung, eine Spontanglukosemessung, eine $\mathrm{HbA}_{\mathrm{lc}}$-Bestimmung und/oder Durchführung eines OGTT erfolgen.

Hohes Risiko für GDM bzw. Risiko für vorbestehende, unerkannte Stoffwechselstörung (Prädiabetes oder Diabetes):

- GDM in einer früheren Schwangerschaft

- Prädiabetes in der Anamnese (gestörte Glukosetoleranz und/oder Nüchternglukose $\geq 100 \mathrm{mg} / \mathrm{dl}$ )

- Kongenitale fetale Fehlbildung in einer früheren Schwangerschaft

- Geburt eines Kindes >4500 g

- Totgeburt

- Habitueller Abortus (>3 Fehlgeburten hintereinander)

- Diabetes-Symptome

- Adipositas $\left(\mathrm{BMI} \geq 30 \mathrm{~kg} / \mathrm{m}^{2}\right)$

- Alter über 35 Jahre

- Metabolisches Syndrom

- Vaskuläre Erkrankung (koronare Herzkrankheit [KHK], Insult, periphere arterielle Verschlusskrankheit [PAVK])

- Familienanamnese von T2DM bei erstgradigen Verwandten

- Ethnizität (arabisch, S- und SO-Asien, Lateinamerika)

Bei Auftreten von diabetesspezifischen Symptomen oder klinischen Auffälligkeiten (vermehrter Durst, Polyurie, Glukosurie; Makrosomie) ist ein Test - auch bei unauffälligem Vorbefund und unabhängig von der Schwangerschaftswoche - unmittelbar durchzuführen (Abb. 1).

In der Austrian Gestational Diabetes Study (AGDS) waren ein GDM in einer früheren Schwangerschaft, das Auftreten einer Glukosurie, Übergewicht (präkon- 
zeptioneller BMI $>27 \mathrm{~kg} / \mathrm{m}^{2}$ ), ein Alter über 30 Jahre und der Verdacht auf Makrosomie im Ultraschall die besten unabhängigen Prädiktoren für einen GDM [5], wobei das Risiko bei vorangegangenem GDM fast 3-fach, ansonsten ungefähr 2-fach erhöht war. Eine frühe Manifestation eines GDM ist v. a. durch eine Adipositas-bedingte Insulinresistenz verursacht [6]. Eine multizentrische europäische Studie zeigte, dass fast jede vierte adipöse Frau bereits vor der 20. Schwangerschaftswoche erhöhte Blutzuckerwerte im Sinne eines GDMs nach IADPSG/WHO 2013-Kriterien und Parameter des metabolischen Syndroms aufwies [7].

Alle Schwangeren müssen in der 24. bis 28. Schwangerschaftswoche mittels eines $75 \mathrm{~g}$ OGTT auf GDM untersucht werden (Abb. 1). Ausgenommen sind Frauen mit bereits vorher diagnostiziertem GDM oder Diabetes bzw. wenn der unmittelbar gemessene Nüchternwert (venöse Plasmaglukose) $92 \mathrm{mg} / \mathrm{dl}$ oder höher ist, da bei diesen Frauen bereits eine Behandlungsbedürftigkeit gegeben ist und auf eine weitere Glukosebelastung verzichtet werden kann. Der OGTT ist im Mutter-Kind-Pass verankert und verpflichtend für den Erhalt des vollen Kinderbetreuungsgelds. Ebenfalls ausgenommen von der Durchführung eines OGTT sollen Frauen nach metabolischer Chirurgie werden, da das Risiko einer postprandialen Hypoglykämie (Dumping-Phänomen) nach der Ingestion der Glukoselösung besonders hoch ist [8]. Nach bariatrischer Operation werden daher regelmäßige Blutzuckerselbstkontrollen zur Diagnose eines GDM herangezogen. Ebenso ist die Verwendung eines Continuous Glucose Monitoring Systems (CGMS) in diesem Fall denkbar [8] (Evidenzklasse C).

\section{Methodik: Diagnostischer $75 \mathrm{~g}$ oraler Glukose- toleranztest (OGTT)}

Der Test soll bei allen Frauen mit bisher unauffälligen oder unbekannten Blutglukosewerten in der Schwangerschaft zwischen 24. und 28. Schwangerschaftswoche morgens nach mindestens achtstündiger Nahrungskarenz durchgeführt werden. Eine Änderung der Ernährung oder Diäten vor dem Test bzw. eine Reduktion der Kohlenhydrate sollten vermieden werden. Ebenso sollten vor dem Test keine außergewöhnlichen körperlichen Belastungen erbracht werden. Der Testbeginn sollte zwischen 6.00 und $9.00 \mathrm{~h}$ erfolgen, da die Glukosetoleranz tageszeitlichen Änderungen unterliegt. Die Schwangere soll die Glukoselösung (75 g Glukose in $300 \mathrm{ml}$ Wasser) innerhalb von $5 \mathrm{~min}$ trinken, während des Testes sitzen (liegende Position vermeiden, keine unnötige körperliche Aktivität) und nicht rauchen. Zur GDM-Diagnostik sollen Blutglukosewerte ausschließlich mit einer qualitätsgesicherten Methode in venösem Plasma direkt gemessen werden oder in venösem Vollblut gemessen und mit einem Faktor von $1,11(+11 \%)$ in venöse Plasmawerte umgerechnet werden.
Tab. 1 Bewertung: OGTT $75 \mathrm{~g}$ (nach WHO- und IADPSGEmpfehlung [10, 48]). OGTT oraler Glukosetoleranztest, WHO „World Health Organization“, IADPSG ,International Association of the Diabetes and Pregnancy Study Groups"

\begin{tabular}{|l|l|}
\hline Zeitpunkt & Venöses Plasma $(\mathrm{mg} / \mathrm{dl})$ \\
\hline Nüchtern & $\geq 92$ \\
\hline $1 \mathrm{~h}$ & $\geq 180$ \\
\hline $\mathrm{h}$ & $\geq 153$ \\
\hline
\end{tabular}

Um möglichst exakte OGTT-Resultate zu erhalten, ist es erforderlich, gewisse Standards zu berücksichtigen [9]. Diese sind wie folgt (abgeleitet nach [9]):

- Messungen aus venösem Plasma und nicht aus Kapillarblut.

- Messung in einem zertifizierten Labor nach zertifizierten Methoden, da eine Einschränkung der Aussagekraft durch präanalytische Fehler sehr wahrscheinlich ist.

- Am Testtag ist vor dem Test eine Einnahme kontrainsulinärer Medikamente (Thyroxin, Progesteron, Glukokortikoide, Sympathomimetika) vor dem OGTT zu vermeiden.

- Nach Einleitung der fetalen Lungenreife mittels Glukokortikoiden sollte man bis zur Testdurchführung mindestens 5 Tage zuwarten.

- Bei Fieber, akuten Erkrankungen oder verordneter Bettruhe ist der Test bis zur vollständigen Genesung zu verschieben.

- Bei operativen Eingriffen am Magen-Darm-Trakt (z.B. bariatrische Operation) ist die Aussagekraft eines OGTT limitiert. Zudem besteht die Gefahr eines Dumping-Syndroms. In diesem Fall sollte eine Blutzuckerselbstmessung über mehrere Tage erfolgen, und Blutzuckerprofile sollten zur Bewertung herangezogen werden.

- Bei Hyperemesis gravidarum oder stärkerer Schwangerschaftsübelkeit ist der Test um einige Tage zu verschieben.

Die internationale Klassifikation (Tab. 1; [10, 11]) beruht auf evidenzbasierten (= aus der HAPO-Studie für kindliche Komplikationen abgeleiteten) Blutzuckergrenzwerten $[12,13]$. Ab einem pathologischen Wert ist ein GDM diagnostiziert.

Anhand von Auswertungen der Schwangerschaftsergebnisse an 5 österreichischen Zentren konnten auch ein einfacher Algorithmus und Risikoscore zur Vorhersage des GDM entwickelt werden, der auf der Messung der Nüchternplasmaglukose beruht und im Einzelfall herangezogen werden kann [14].

\section{Prävention}

Die Prävention von GDM wurde in zahlreichen Studien untersucht und dabei an verschiedenen Risikogruppen getestet. Bei Frauen mit Adipositas konnte in den bisherigen großen Studien mit Lebensstilintervention weder eine Verbesserung im fetalen Outcome 
Abb. 2 Flussdiagramm Gestationsdiabetes (GDM) Behandlung. BG Blutglukose, $R R$ Blutdruck, OGTT oraler Glukosetoleranztest

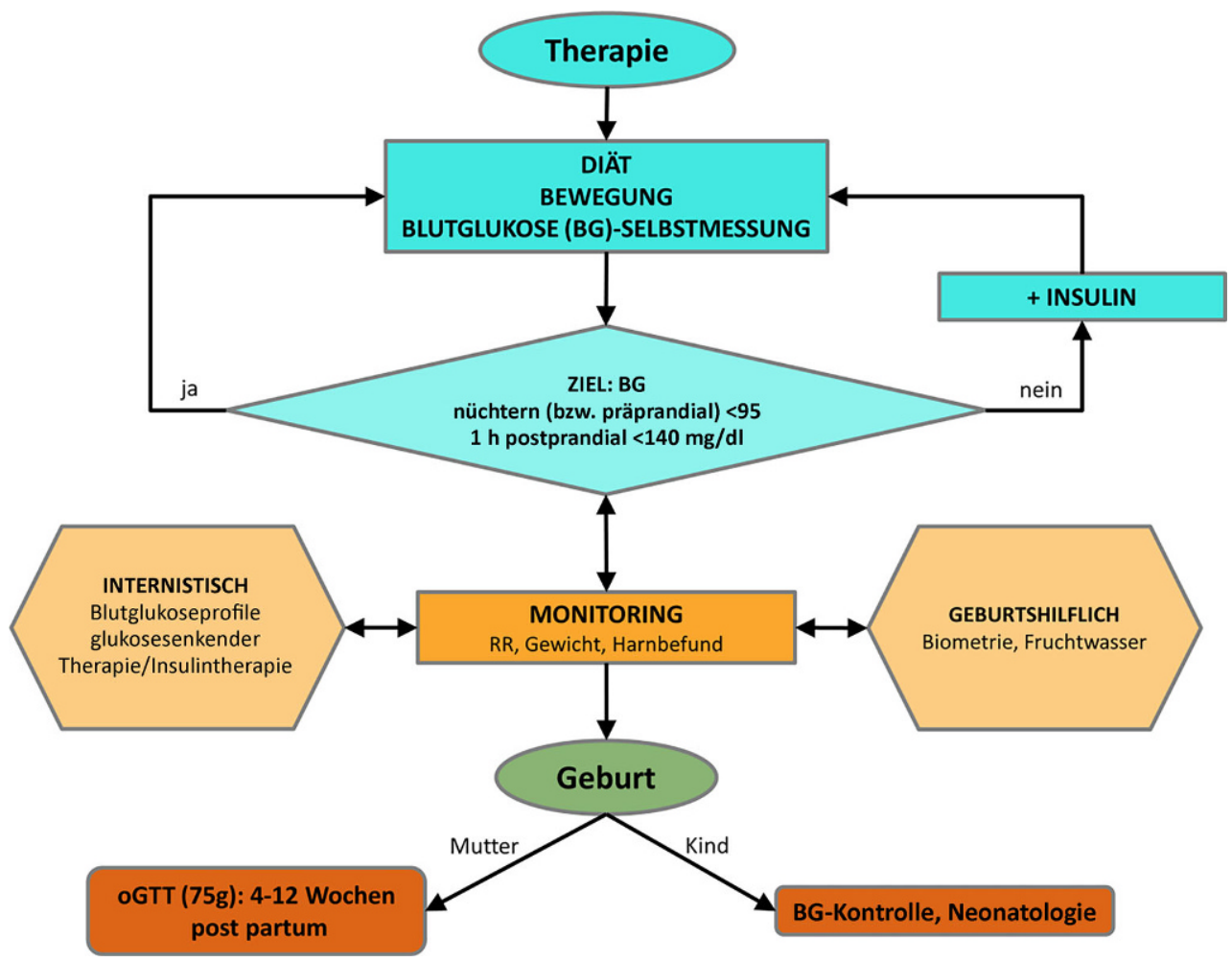

(„large for gestational age“, LGA oder Makrosomie) noch eine Verbesserung der mütterlichen Stoffwechselsituation oder GDM-Prävalenz erreicht werden [15]. In der DALI-Studie konnte im Vergleich 3 verschiedener Interventionsgruppen (gesunde Ernährung, körperliche Aktivität, Kombination aus beiden) eine signifikante Gewichtsabnahme in der kombinierten Interventionsgruppe im Vergleich zur Kontrolle gezeigt werden, dies hatte aber keinen Einfluss auf die mütterlichen oder kindlichen Outcomes bei Geburt [15]. Unklare Evidenz liegt bei Interventionen durch körperliche Aktivität vor. Bei adipösen Frauen konnten mit Ernährungsmaßnahmen das GDMRisiko sowie eine kindliche Makrosomie verringert werden. Dies konnte bei körperlicher Aktivität nicht beobachtet werden. Eine Supplementation mit Probiotika und Myo-Inositol konnte das GDM-Risiko verringern. Bei adipösen schwangeren Frauen konnte unter Gabe von Metformin keine Reduktion des GDM-Risikos und keine Verbesserung des mütterlichen Stoffwechsels oder Geburtsoutcomes erreicht werden [15]. Die bisherigen Studien zeigen, dass der Beginn einer Lebensstilmodifikation nach dem ersten Trimester zu spät ist. Dies wird in einer Übersichtsarbeit bestätigt, die zeigt, dass ein Interventionsbeginn vor der Schwangerschaft effektiver ist als in der frühen Schwangerschaft [15].

\section{Therapie}

\section{1) Diabetologische/internistische Betreuung}

Erstellung eines individuellen Therapieplans bestehend aus einer Lebensstilmodifikation mit Ernährungsempfehlungen, Bewegung und Blutglukoseselbstmessungen (Abb. 2):

a) Ernährung: Je nach Körpergewicht und körperlicher Aktivität ausgerichteter Diätplan (bei Normalgewicht ca. 24-30 kcal/kg: 40-50\% Kohlenhydrate, 30-35 \% Fett und 20 \% Eiweiß). Auf schnell resorbierbare Kohlenhydrate sollte verzichtet werden. Eine ballaststoffreiche Ernährung (ca. $30 \mathrm{~g} / \mathrm{Tag}$ ) ist zu empfehlen. Die ausreichende Versorgung mit Mineralstoffen und Vitaminen ist $\mathrm{zu}$ berücksichtigen (Eisen, Folsäure, Vitamin D, Kalzium, Vitamin B, Magnesium, Jod). Hier gelten die nationalen Referenzwertempfehlungen für Nahrungszufuhr in der Schwangerschaft (D-A-CH Referenzwerte, www. oege.at) Die täglich empfohlene Proteinzufuhr in der Schwangerschaft entspricht der einer gesunden Schwangerschaft (60-80 g/Tag). Eine Aufteilung der Mahlzeiten auf 3 kleine bis mittlere Hauptmahlzeiten und 2 bis 4 kleine Zwischenmahlzeiten inklusive Abendsnack sollte erfolgen [16]. Die Endocrine Society empfiehlt bei Adipositas eine Kalorienrestriktion um etwa ein Drittel, so keine deutliche Gewichtsreduktion (bis maximal $5 \mathrm{~kg}$ ) und Katabolismus auftritt. Die minimale Aufnahme liegt zwischen 1600 und $1800 \mathrm{kcal} / \mathrm{Tag}$ [16]. Eine Gewichts- 
Tab. 2 Gewichtszunahme in der Schwangerschaft nach IOM-Empfehlungen [17]

\begin{tabular}{|c|c|c|c|}
\hline BMI & BMI-Limits $\left(\mathrm{kg} / \mathrm{m}^{2}\right)(\mathrm{WHO})$ & $\begin{array}{l}\text { Empfohlene Zunahme während } \\
\text { der Schwangerschaft (kg) }\end{array}$ & $\begin{array}{l}\text { Empfohlene Gewichtszunahme/Woche (kg/Woche) } \\
(2 .+3 \text {. Trimenon) }\end{array}$ \\
\hline Untergewicht & $<18,5$ & $13-18$ & 0,51 \\
\hline Normalgewicht & $18,5-24,9$ & $11-16$ & 0,42 \\
\hline Übergewicht & $25,0-29,9$ & $7-11$ & 0,28 \\
\hline Adipositas & $\geq 30,0$ & $5-9$ & 0,22 \\
\hline
\end{tabular}

Tab. 3 Einstellungsziele

\begin{tabular}{|l|l|}
\hline Zeitpunkt & Kapilläres Vollblut (mg/dl) \\
\hline Nüchtern (präprandial) & $65-95$ \\
\hline h h postprandial & $<140$ \\
\hline $2 \mathrm{~h}$ postprandial & $<120$ \\
\hline
\end{tabular}

kontrolle muss bei jedem Kontrollbesuch erfolgen bzw. selbstständig wöchentlich von der Patientin dokumentiert werden.

Die Gewichtszunahme in der Schwangerschaft sollte dabei den Empfehlungen des Institute of Medicine folgen (Tab. 2; [17]).

b) Schulung in Blutglukoseselbstmessung (BGSM). Dokumentation der BG-Profile: mindestens 4 Messungen täglich (nüchtern, $1 \mathrm{~h}$ [evtl. auch $2 \mathrm{~h}$ ] postprandial). Bei Beginn einer Insulintherapie sollte die Patientin über die Symptome und das Risiko von Hypoglykämien sowie über das richtige Verhalten in dieser Situation von geeignetem Fachpersonal geschult werden. Eine schlechte Adhärenz zu regelmäßigen selbstständigen Blutzuckermessungen steht mit einem erhöhten Präeklampsierisiko in Zusammenhang und war in einer Studie mit Zugehörigkeit zu niedriger sozialer Klasse, nichteuropäischer Herkunft und Diabetes in der Familienanamnese assoziiert [18]. Hingegen konnten bei guter Adhärenz mit guter Blutzuckereinstellung keine Unterschiede zwischen täglich 4 Messungen und Messungen jeden zweiten Tag in mütterlichen und kindlichen Outcomes festgestellt werden [19]. Eine Reduktion der Blutzuckermessungen (Messung alle 2 Tage) kann bei guter Blutzuckereinstellung und fortgeschrittener Schwangerschaft überlegt werden.

c) Bewegung: Bei einer unproblematischen Schwangerschaft ist regelmäßige moderate körperliche Aktivität ein weiterer Bestandteil des Therapiekonzepts. Die Aktivitätszeit sollte dabei mindestens 150 min pro Woche betragen und sollte in den Alltag integriert werden. Bei Ausübung von Sport sollten Sportarten gewählt werden, die mit einer Schwangerschaft vereinbar sind (kein Kontaktsport, Kampfsport, Sportarten mit hoher Sturz- oder Verletzungsgefahr) und dem jeweiligen Trainingszustand entsprechen. d) Therapieziele und pharmakologische Therapie: Bei unzureichender Einstellung durch Lebensstilmaßnahmen ist unmittelbar eine medikamentöse Therapie einzuleiten. Insulin sollte gegenüber oralen glukosesenkenden Medikamenten aufgrund der deutlich besseren Studienlage und keiner Plazentagängigkeit bevorzugt eingesetzt werden [20]. Werden die Grenzwerte überschritten (Tab. 3), ist eine individuell anzupassende Insulintherapie zu beginnen. Liegen Nüchternglukosewerte über $110 \mathrm{mg} /$ $\mathrm{dl}$ ist ein sofortiger Therapiebeginn mit Insulin empfehlenswert [9]. Die mütterlichen BG-Profile müssen auch während der Geburt im Zielbereich liegen $(80-130 \mathrm{mg} / \mathrm{dl})$, um neonatale Hypoglykämien und Anpassungsstörungen zu vermindern. Der $\mathrm{HbA}_{1 c}$-Wert ist für die Diagnose eines GDM ungeeignet, kann aber zur Verlaufskontrolle der Metabolik herangezogen werden und soll jedenfalls in einem Referenzbereich von $\mathrm{HbA}_{1 \mathrm{c}}<6 \%$ angesiedelt sein.

Bei wiederholten Glukosewerten zwischen 90 und $95 \mathrm{mg} / \mathrm{dl}$ nüchtern/präprandial und/oder 130 und $140 \mathrm{mg} / \mathrm{dl} 1 \mathrm{~h}$ postprandial soll die fetale Biometrie zur Entscheidung, ob eine medikamentöse Therapie begonnen werden muss, herangezogen werden. Liegt eine fetale asymmetrische Wachstumssteigerung vor und ist die abdominelle Zirkumferenz über der 75. Perzentile des Gestationsalters, ist eine Insulinisierung zu empfehlen bzw. die Insulindosis zu steigern. Bei kindlicher Makrosomie oder Wachstumsretardierung können auch individuell angepasste gering niedrigere oder höhere mütterliche Blutglukosegrenzwerte gewählt werden [9].

Regelmäßige biometrische Kontrollen sollen individuell, den Bedürfnissen der schwangeren Frauen entsprechend, im Abstand von wenigen Tagen bis 3 Wochen erfolgen. Dabei ist anhand der Blutglukoseprofile eine Therapieanpassung (Insulindosis) je nach Erfordernis durchzuführen. Der Blutdruck und die Gewichtszunahme sollten kontrolliert und ein Harnbefund durchgeführt werden.

Insulin

Für Insulin ist die derzeit vorliegende Datenlage am besten dokumentiert. Primär wird NPH-Insulin als Basisinsulin verwendet. Auch andere Langzeitinsuline (Glargin, Glargin U300 oder Detemir) können ohne Bedenken in der Schwangerschaft angewendet werden, jedoch zeigt eine rezente Metaanalyse kei- 
ne signifikanten Differenzen in Bezug auf mehrere maternale oder neonatale Parameter im Vergleich zu NPH-Insulin [21]. Im Vergleich NPH zu Glargin gibt es keine Unterschiede im Geburtsgewicht sowie vergleichbares Risiko für neonatale Komplikationen und Malformationen. Ebenso sind die mütterlichen Outcomes Präeklampsie und Schwangerschaftshypertonie vergleichbar selten. Für Insulin Detemir ist das Risiko für LGA oder neonatale Hypoglykämie ebenso vergleichbar mit NPH-Insulin [21]. Für neuere Langzeitinsuline (z.B. Degludec) gibt es noch keine Evidenz. Randomisiert kontrollierte Studien zu Insulin Degludec werden erwartet, Fallbeschreibungen zeigen derzeit keine maternale oder kindliche Komplikationen in der Schwangerschaft [22].

Schnell wirksame Insuline werden zur Korrektur postprandialer Spitzen angewendet. Vielfach finden Insulin Lispro oder Aspart Anwendung und sollten gegenüber Humaninsulin auch aufgrund der einfacheren Handhabung vorgezogen werden. $\mathrm{Zu}$ Glulisin liegen derzeit nur Vigilanzdaten in der Gravidität vor [23], die keine besonderen Auffälligkeiten in der Schwangerschaft zeigen, jedoch sollte es derzeit aufgrund der unzureichenden Datenlage nicht verwendet werden. Die Analoga konnten aber bisher nur teilweise Überlegenheit gegenüber Normalinsulin zeigen [21]. Der Vergleich von Aspart zu Humaninsulin zeigt keine Unterschiede bei Makrosomie oder Häufigkeit von Kaiserschnittgeburten. Lispro verglichen $\mathrm{zu} \mathrm{Hu}$ maninsulin war mit niedrigerer Inzidenz für Ikterus und weniger maternalen Hypoglykämien verbunden, andererseits wurden in der Gruppe mit Lispro höhere Inzidenzen für LGA und höheres Geburtsgewicht berichtet. Das ultraschnell wirksame Insulin Aspart (Fiasp) ist in der Schwangerschaft zugelassen. Fiasp wird rascher resorbiert, ist daher schneller wirksam als bisherige Analoginsuline und wird zur Optimierung postprandialer Hyperglykämien angewendet.

\section{Orale Antidiabetika}

Der Sulfonylharnstoff Glibenclamid und das Biguanid Metformin werden in manchen Therapieempfehlungen (z.B. NICE, ADA Guidelines, DDG-Leitlinien) als mögliche Alternativen oder zusätzlich zu Insulin in der Schwangerschaft genannt. Metformin (FDA Kategorie B) und Glibenclamid sind plazentagängig. Randomisierte kontrollierte Untersuchungen über den Einsatz von Glibenclamid und Metformin (beide Evidenzklasse Ib, beide plazentagängig) [24, 25] bei GDM zeigten keine wesentlichen Unterschiede zwischen der oralen Behandlung und einer Insulintherapie. Bei Verwendung eines dieser Präparate in der Schwangerschaft sollten die Patientinnen in die Therapieentscheidung mit einbezogen werden.

Metformin An die Gabe von Metformin sollte insbesondere bei übergewichtigen insulinresistenten Frauen als Monotherapie oder in Kombination mit Insulin gedacht werden [16]. Unter Gabe von Metformin ab der 20. Schwangerschaftswoche wurde eine niedrigere Rate schwerer neonataler Hypoglykämien, jedoch eine höhere Frühgeburtenrate beobachtet [24]. Eine rezente Metaanalyse zeigt geringere maternale Gewichtszunahme in der Schwangerschaft und häufiger Geburten vor dem Geburtstermin bei Frauen mit Metformintherapie verglichen zu Insulintherapie [26]. Die Mütter in der Metformingruppe konnten bei der Nachuntersuchung postpartal eher ihr Ausgangsgewicht erreichen als die insulinbehandelten Frauen; bezüglich des postpartalen Glukosetoleranzstatus bestanden keine Unterschiede [24]. Ein weiterer Grund für einen zögerlichen Einsatz von Metformin sind fehlende Langzeitdaten zur kindlichen Entwicklung. Die MIG-Studie zeigte, dass Kinder aus der Metformintherapiegruppe in der Schwangerschaft erhöhte subkutane Fettmasse verglichen zur Insulingruppe aufwiesen - die Gesamtkörperfettmasse blieb jedoch vergleichbar [27]. Eine rezente Studie konnte bei Nachkommen von Müttern, die bei PCOS 1700-2000 mg Metformin in der Schwangerschaft erhielten, 4 Jahre nach Entbindung ein deutlich erhöhtes Risiko für Übergewicht und Adipositas im Vergleich zur Placebogruppe feststellen [28].

Sulfonylharnstoff Bei Ablehnung einer notwendigen Insulintherapie stellt eine Therapie mit Glibenclamid eine mögliche, wenn auch hierzulande äußerst selten genutzte Alternative zur Behandlung eines GDM dar. Eine Insulintherapie sollte aber allenfalls präferiert werden, wenn der GDM bereits vor der 25. Schwangerschaftswoche besteht oder Glukosewerte über $110 \mathrm{mg} / \mathrm{dl}$ vorherrschen [16] Vorteile von Metformin gegenüber Glibenclamid konnten in einer Metaanalyse gezeigt werden - unter Glibenclamidtherapie konnten höhere maternale Gewichtszunahme sowie vermehrte Raten von fetaler Makrosomie und neonataler Hypoglykämie festgestellt werden [26]. Auch im Vergleich zu Insulin häufte sich unter Glibenclamid das Risiko für Makrosomien, neonatalen Hypoglykämien und höherem Geburtsgewicht [26]. Neugeborene aus Glibenclamid behandelten GDMSchwangerschaften weisen zudem höheres Risiko für Geburtskomplikationen (Hypoglykämie, Atemnotsyndrom des Neugeborenen [RDS], Intensivstationsaufenthalte, LGA) auf [29]. In einer rezenten randomisiert kontrollierten Studie konnte ein höheres Risiko an kumulativ perinatalen Komplikationen (Makrosomie, Hypoglykämie, Hyperbilirubinämie) in der Glibenclamidgruppe im Vergleich zu Insulin festgestellt werden [30]. Zwar konnten keine erhöhten Makrosomieraten im Vergleich Glibenclamid zu Insulin beobachtet werden, jedoch waren die Hypoglykämieraten bei geringen Glibenclamiddosen von durchschnittlich $5,4 \mathrm{mg}$ signifikant höher als unter Insulin. Aufgrund dieser Datenlage ist die primäre Verwendung von Insulin zur Behandlung von GDM klar $\mathrm{zu}$ favorisieren [31]. 
Abb. 3 Flussdiagramm Blutzuckermanagement bei Risikoneugeborenen. BZ Blutzucker, MD Maltodextrin, pp postprandial, iv intravenös

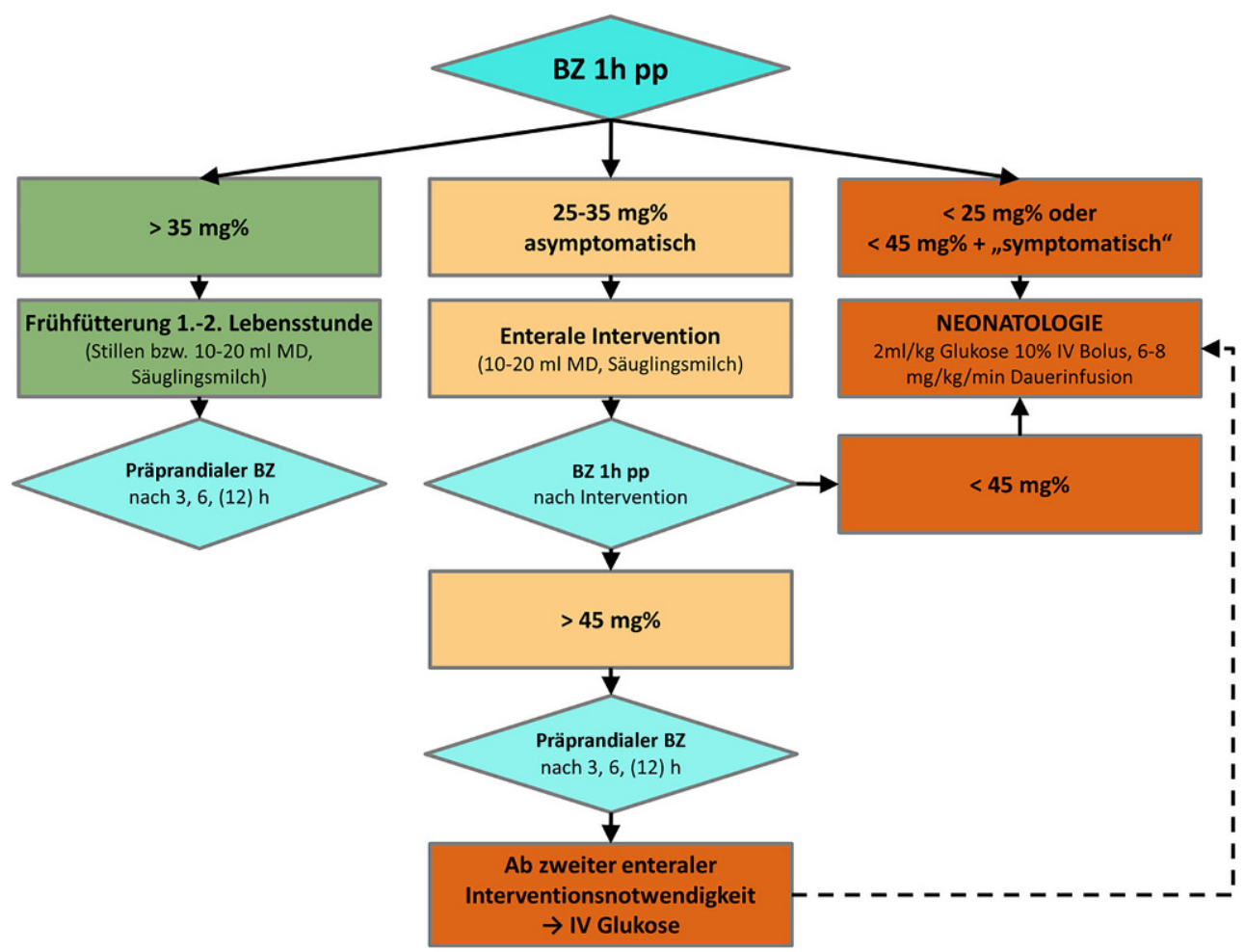

\section{2) Geburtshilfliche Überwachung}

- Ein- bis dreiwöchentliche klinische Kontrollen

- Bei Hyperglykämie in Frühschwangerschaft: frühes Organscreening durch Ultraschall zum Ausschluss von Fehlbildungen (v. a. Herz, Niere)

- Ultraschall (Biometrie, Fruchtwasser, evtl. Doppler), Wachstumskurven (v.a. Wachstumszunahme des Abdomens = asymmetrische Wachstumszunahme; Polyhydramnion) beachten

- Achten auf erhöhtes Risiko zur Entwicklung einer Schwangerschaftshypertonie, Präeklampsie, Infektionen

- Idealen Geburtstermin und Geburtsmodus festlegen

Schwangere mit GDM sollten in einem Krankenhaus mit diabetologischer Erfahrung und angeschlossener Neonatologie entbunden werden. Eine Überschreitung des Geburtstermins sollte bei Schwangeren mit insulinpflichtigem GDM vermieden werden.

Ob zwischen Schwangerschaftswoche $38+0$ und $40+0$ eine Geburtseinleitung stattfinden soll, soll individuell entschieden werden. Dabei sollen der Insulinbedarf, die Ultraschallbefunde (Kindsgewicht, Doppler, Fruchtwasser), maternale Erkrankungen wie Präeklampsie und die vorausgegangenen Schwangerschaftsverläufe in die Entscheidung mit einbezogen werden [32-34]. Eine Einleitung wegen schlechter Blutzuckereinstellung vor Schwangerschaftswoche $38+0$ sollte wegen frühgeburtlichkeitsbedingter Mor-

bidität vermieden werden. Vielmehr sollte eine pränatale Optimierung der Blutzuckerwerte erfolgen.

Es ist bekannt, dass das Risiko für eine Schulterdystokie ab einem Geburtsgewicht von $4250 \mathrm{~g}$ signifikant ansteigt [35]. Ab einem geschätzten Geburtsgewicht von $4500 \mathrm{~g}$ sollte deshalb bei einer Schwangeren mit GDM eine Sectio empfohlen werden. Bei einem Schätzgewicht von 4000-4499g sollte eine differenzierte Aufklärung der Schwangeren über individuell erhöhtes Schulterdystokierisiko erfolgen, insbesondere bei ausgeprägter Kopf-Abdomen-Differenz.

\section{3) Überwachung und Management des Neugeborenen}

Ein Routinemonitoring ist für eine Hochrisikopopulation an Neugeborenen sinnvoll (Abb. 3), zu denen Kinder aus diabetischen Schwangerschaften bzw. solche, die aus einem anderen Grund einem erhöhten Risiko für die Entwicklung einer Hypoglykämie ausgesetzt sind, zählen (z. B. dystrophe Neugeborene; LGABabys).

Generell zu vermeiden sind prolongierte und rezidivierende Hypoglykämien, da diese mit akuten systemischen und neurologischen Langzeitkomplikationen einhergehen können [36].

\section{Blutglukosebestimmungen nach Geburt}

Erste Messung: In Abhängigkeit vom Risikofaktor soll bei zu erwartender Hypoglykämie 30-60 min nach Geburt (bei schlecht eingestellter Gestationsdiabetes), ansonsten innerhalb der ersten 2 Lebensstunden 
gemessen werden. Der Einfachheit halber kann aber für die gesamte Population der Zeitpunkt $1 \mathrm{~h}$ nach Geburt festgelegt werden. Eine Ausnahme ist hier nur die Indikation schlecht eingestellter Schwangerschaftsdiabetes der Mutter - hier sollte die erste Messung eher bereits nach 30 min erfolgen.

Weitere Messungen: Zumindest 2-mal vor den nächsten beiden Mahlzeiten (ca. nach 3 und $6 \mathrm{~h}$, evtl. auch nach $12 \mathrm{~h}$ z.B. bei mütterlichem Diabetes, grenzwertigen Messungen). Ende der Messungen: Es sollen zumindest 2 normale präprandiale Glukosewerte hintereinander dokumentiert sein, um die Messungen beenden zu können.

Messung nach Intervention: Bei enteraler oder intravenöser Intervention aufgrund einer Hypoglykämie erfolgt eine Kontrolle $1 \mathrm{~h}$ nach Intervention.

Die Bestimmung der Blutglukose muss unmittelbar nach der Blutabnahme erfolgen. Bei Verwendung von Schnelltests (Glukometer) weisen diese im hypoglykämischen Bereich unter $45 \mathrm{mg} / \mathrm{dl}$ Glukose in Abhängigkeit vom Hersteller Ungenauigkeiten auf. Ein mit dieser Messmethode ermittelter hypoglykämischer Wert sollte durch eine laborchemische Bestimmung kontrolliert werden. Dies sollte aber zu keiner Verzögerung der Therapie führen.

\section{Interventionsgrenzen und therapeutische Zielwerte}

Aufgrund interindividueller Schwankungen gibt es keine absoluten Grenzwerte für die Behandlung der Hypoglykämie des Neugeborenen. Vorgeschlagen werden pragmatische „Interventionsgrenzen“ bei denen eine Intervention in Erwägung gezogen werden sollte (siehe Intervention: $<25 \mathrm{mg} \%$ intravenös, 25-35 mg\% enteral).

Die „therapeutischen Zielwerte“ beinhalten einen Sicherheitsabstand.

\section{Ernährung des Säuglings nach Geburt}

Insbesondere Kinder aus diabetischer Schwangerschaft sollen bereits innerhalb der ersten Lebensstunde angelegt werden. Nahrung aus der Flasche (Anfangsmilch) soll nur angeboten werden, wenn Stillen nicht möglich/erwünscht ist bzw. als Intervention bei zu niedrigem Blutzucker (s. „Intervention“) [37].

\section{Intervention}

Enteral: Nur bei asymptomatischer Hypoglykämie 25-35 mg\% $\rightarrow$ Verabreichung von 10-20 ml Säuglingsmilch oder Maltodextrinlösung $15 \%$. Eine Verabreichung von reiner Glukoselösung wird ausdrücklich nicht empfohlen.

Intravenös: Bei extremer Hypoglykämie $<25 \mathrm{mg} \%$, symptomatischen Kindern $<45 \mathrm{mg} \%$ oder persistierender Hypoglykämie (falls die Kontrolle $1 \mathrm{~h}$ nach Intervention $<45 \mathrm{mg} \%$ ist, oder falls trotz zweimaliger enteraler Intervention weiter korrekturbedürftige präprandiale Blutzuckerwerte gemessen werden) $\rightarrow 2 \mathrm{ml} / \mathrm{kg}$ Glukose $10 \%$ als i.v.-Bolus, gefolgt von
6-8 $\mathrm{mg} / \mathrm{kg} / \mathrm{min}$ als kontinuierliche Infusion. Es wird eine schrittweise Reduktion der intravenösen Glukosezufuhr unter Beginn der enteralen Ernährung und präprandialen Blutzuckerkontrolle empfohlen.

Kinder von Frauen mit GDM haben ein höheres Risiko, im späteren Leben übergewichtig zu werden und ein metabolisches Syndrom bis hin zu einem Diabetes zu entwickeln [38]. Deshalb sollte bei allen - und besonders bei makrosomen - Kindern auf eine normale Gewichtsentwicklung geachtet werden (s. „Nachbetreuung der Kinder").

\section{Nachbetreuung der Mutter}

Falls nach der Geburt normale Blutzuckerwerte erhoben werden (nüchtern $<100 \mathrm{mg} / \mathrm{dl}$ und unabhängig von Mahlzeiteneinnahme $<200 \mathrm{mg} / \mathrm{dl}$ ) ist keine weitere Diättherapie oder Blutzuckerselbstmessung notwendig. Allerdings muss 4 bis 12 Wochen nach der Geburt eine Reklassifizierung der mütterlichen Glukosetoleranz mittels Standard-OGTT (2h-75g OGTT) erfolgen. Bei pathologischem Befund müssen Therapieempfehlungen erfolgen (s. allgemeine Lebensstilintervention, Diabetestherapie). Im Fall eines postpartal bestehenden Prädiabetes (gestörte Glukosetoleranz [2-h-Wert 140-199 mg/dl] im OGTT oder erhöhter Nüchternglukose [100-125 mg/dl]) ist eine Diät zu verordnen und vermehrte körperliche Aktivität (Ausdauertraining) anzuraten. Eine Subanalyse des Diabetes Prevention Programs zeigte, dass bei vergleichbarer Ausgangslage bezüglich Glukosetoleranzstatus und Insulinresistenz Frauen mit Anamnese eines GDM ein doppelt so hohes Risiko für die Progression zu einem manifesten Diabetes aufwiesen wie jene Frauen, die eine unauffällige Schwangerschaft hatten und dass diese Gruppe von einer Therapie mit Metformin besonders profitierte [39]. Diese wurde im 10Jahres-Follow-up erneut bestätigt: Lebensstilmaßnahmen und Metformin konnten das Diabetesrisiko um 35-40 \% verglichen zu Placebo verringern [40]. Eine Analyse des Wiener GDM-Programms zeigte dass ein 2-h-Blutzuckerwert im ersten OGTT post partum über $140 \mathrm{mg} / \mathrm{dl}$, ein HDL unter $50 \mathrm{mg} / \mathrm{dl}$ und ein Alter über 35 Jahre die wichtigsten unabhängigen Risikofaktoren für die Entwicklung eines manifesten Diabetes innerhalb von 10 Jahren darstellten [41].

Alle Patientinnen müssen außerdem über ihr erhöhtes Risiko für die Entwicklung eines T2DM, eines GDM-Rezidivs (20-50 \%) bei neuerlicher Schwangerschaft, ein erhöhtes kardiovaskuläres Risiko sowie über Möglichkeiten der Diabetesprävention informiert werden.

Bei unauffälligem Erstbefund sollen die Frauen alle 2 bis 3 Jahre mittels OGTT oder zumindest mittels Messung der Nüchternglukose und des $\mathrm{HbA}_{1 \mathrm{c}}$ nachuntersucht werden (WHO-Kriterien).

Diabetische Schwangere sollen, wenn immer es möglich ist, ihr Kind stillen, da protektive Effekte in Studien gezeigt werden konnten [16]. Bei einer 
Stilldauer von mehr als 3 Monaten weisen stillende Mütter eine um bis zu 10 Jahre verzögerte Progression von GDM zu T2DM auf als nicht stillende Frauen [42].

Eine bestehende Metformintherapie bei stillenden diabetischen Müttern sollte fortgeführt werden, falls dies erforderlich ist [16].

Frauen nach GDM sollen reine Gestagenpräparate insbesondere in der Stillzeit vermeiden, da sich dadurch das Risiko für die Manifestation eines T2DM erhöhen könnte [43]. Außer auf eine Glukosestoffwechselstörung soll auch auf weitere kardiovaskuläre Risikoparameter wie Dyslipidämie und Hypertonie untersucht werden, da Frauen nach GDM ein höheres kardiovaskuläres Risiko aufweisen [44].

\section{Nachbetreuung der Kinder}

Bei Nachkommen von GDM-Schwangerschaften ist ein erhöhtes Risiko für Übergewicht/Adipositas und T2DM bekannt [45]. Ein gesunder Lebensstil und regelmäßige Gewichtskontrollen sind zu empfehlen. Bei Hinweisen auf Hyperglykämie ist eine sofortige Abklärung empfohlen (cave: keine $\mathrm{HbA}_{\mathrm{lc}}$-Testung bei Kindern und Jugendlichen zur Diagnosestellung [s. auch Leitlinie Definition, Diagnosescreening]). Ein T2DMScreening sollte bei asymptomatischen Kindern und Jugendlichen mit Adipositas (BMI > 95. Perzentile, geschlechts- und altersadjustiert) oder mit Übergewicht (BMI > 85. Perzentile) und mütterlichem GDM in der Schwangerschaft des Kindes erfolgen [46].

\section{Evidenzlage}

Gesichert ist, dass eine mütterliche Hyperglykämie im 1. Trimenon mit einem höheren Risiko für die Entwicklung einer diabetischen Embryopathie, im 2. und 3. Trimenon für die Entwicklung einer diabetischen Fetopathie mit erhöhter Morbidität und Mortalität assoziiert ist [2]. Die „Hyperglycemia and adverse pregnancy outcome (HAPO)“ Studie zeigte des Weiteren, dass ein kontinuierlicher Zusammenhang zwischen der Höhe der mütterlichen Blutzuckerwerte im OGTT und den kindlichen Komplikationen besteht [12].

Während eine Diättherapie alleine nicht eindeutig zu besseren fetalen Ergebnissen in Metaanalysen führt, ist die Verbesserung der postprandialen Blutglukosewerte unter Insulintherapie mit einer geringeren Morbidität verbunden. Neue Studien konnten belegen, dass eine Behandlung (Diät, Insulin bei Bedarf) des GDM das Risiko für schwere kindliche Komplikationen im Vergleich zu unbehandelten Frauen signifikant reduzieren konnte [3, 4].

Es konnte klar gezeigt worden, dass Frauen nach GDM ein besonders hohes Risiko für die Entwicklung eines T2DM haben [1] und auch ein höheres Risiko für folgende kardiovaskuläre Erkrankungen vorliegt [47]. Lebensstiländerungen im Sinne der Diabetesprävention führen zu einer deutlichen Verringerung der Diabetesmanifestationsrate [39, 40]. In der Schwangerschaft war v. a. in Risikogruppen der Erfolg der bisherigen GDM-Präventionsstudien bescheiden oder nicht vorhanden [15]. Kinder von Frauen mit GDM haben ein höheres Risiko, selbst übergewichtig zu werden und Stoffwechselstörungen zu entwickeln.

Funding Open access funding provided by Medical University of Vienna.

Interessenkonflikt A. Kautzky-Willer hat von folgenden Unternehmen, die teils auch fördernde Mitglieder der ÖDG sind, Forschungsunterstützungen und/oder Honorare erhalten: AstraZeneca, Bristol-Myers Squibb, GlaxoSmithKline Pharma, Eli Lilly, Merck Sharp \& Dohme, Novartis, Novo Nordisk, Roche, Sanofi-Aventis. J. Harreiter hat von folgenden Unternehmen, die teils auch fördernde Mitglieder der ÖDG sind, Forschungsunterstützungen und/oder Honorare erhalten: AstraZeneca, Novo Nordisk, Takeda. Y. WinhoferStöckl hat von folgenden Unternehmen, die auch fördernde Mitglieder der ÖDG sind, Forschungsunterstützungen/ Honorare erhalten: Sanofi Aventis, Eli Lilly, AstraZeneca, Novartis. M. Lechleitner hat von folgenden Unternehmen, die auch fördernde Mitglieder der ÖDG sind, Honorare erhalten: AstraZeneca, Bristol-Myers Squibb, Germania Pharmazeutika, GlaxoSmithKline Pharma, Eli Lilly, Medtronic, Merck Sharp \& Dome, Novartis, Novo Nordisk, Pfizer, Sanofi-Aventis, Takeda. R. Weitgasser hat von folgenden Unternehmen, die teils auch fördernde Mitglieder der ÖDG sind, Forschungsunterstützungen und/oder Honorare erhalten: Abbott, Allergan, AstraZeneca, Boehringer-Ingelheim, Eli Lilly, Merck Sharp \& Dohme, Novo Nordisk, Roche, SanofiAventis, Servier, Takeda. D. Bancher-Todesca, A. Berger und A. Repa geben an, dass kein Interessenkonflikt besteht.

Open Access Dieser Artikel wird unter der Creative Commons Namensnennung 4.0 International Lizenz (http:// creativecommons.org/licenses/by/4.0/deed.de) veröffentlicht, welche die Nutzung, Vervielfältigung, Bearbeitung, Verbreitung und Wiedergabe in jeglichem Medium und Format erlaubt, sofern Sie den/die ursprünglichen Autor(en) und die Quelle ordnungsgemäß nennen, einen Link zur Creative Commons Lizenz beifügen und angeben, ob Änderungen vorgenommen wurden.

\section{Literatur}

1. Bellamy L, Casas JP, Hingorani AD, et al. Type 2 diabetes mellitus after gestational diabetes: a systematic review and meta-analysis. Lancet. 2009;373(9677):1773-9. https://doi. org/10.1016/S0140-6736(09)60731-5.

2. Reece EA, Leguizamon G, Wiznitzer A. Gestational diabetes: the need for a common ground. Lancet. 2009;373(9677):1789-97. https://doi.org/10.1016/S01406736(09)60515-8.

3. Crowther CA, Hiller JE, Moss JR, et al. Effect of treatment of gestational diabetes mellitus on pregnancy outcomes. N Engl J Med. 2005;352(24):2477-86. https://doi.org/10. 1056/NEJMoa042973.

4. Alwan N, Tuffnell DJ, West J. Treatments for gestational diabetes. Cochrane Database Syst Rev. 2009; https://doi. org/10.1002/14651858.CD003395.pub2.

5. Kautzky-Willer A, Bancher-Todesca D, Weitgasser R, et al. The impact of risk factors and more stringent diagnostic criteria ofgestational diabetes on outcomes in centralEuropean women. J Clin Endocrinol Metab. 2008;93(5):1689-95. https://doi.org/10.1210/jc.2007-2301. 
6. Bozkurt L, Göbl CS, Pfligl L, et al. Pathophysiological characteristics and effects of obesity in women with early and late manifestation of gestational diabetes diagnosed by the International Association of Diabetes and Pregnancy Study Groups criteria. J Clin Endocrinol Metab. 2015;100(3):1113-20. https://doi.org/10.1210/jc. 2014-4055.

7. Harreiter J, Simmons D, Desoye G, et al. IADPSG and WHO 2013 gestational diabetes mellitus criteria identify obese women with marked insulin resistance in early pregnancy. Diabetes Care.2016;39(7):e90-e2.https://doi.org/10.2337/ dc16-0200.

8. Harreiter J, Schindler K, Bancher-Todesca D, et al. Management of pregnant women after bariatric surgery. J Obes. 2018;2018:4587064. https://doi.org/10.1155/2018/ 4587064.

9. Deutsche Diabetes Gesellschaft, Deutsche Gesellschaft für Gynäkologie und Geburtshilfe. S3-Leitlinie Gestationsdiabetes mellitus (GDM), Diagnostik, Therapie und Nachsorge. 2. Aufl.AWMF-Registernummer 057-008.2018.

10. Metzger BE, Gabbe SG, Persson B, et al. International association of diabetes and pregnancy study groups recommendations on the diagnosis and classification of hyperglycemia in pregnancy. Diabetes Care. 2010;33(3):676-82. https://doi.org/10.2337/dc09-1848.

11. World Health Organization. Diagnostic criteria and classification of hyperglycaemia first detected in pregnancy: a World Health Organization guideline. Diabetes Res Clin Pract. 2014;103(3):341-63. https://doi.org/10.1016/j. diabres.2013.10.012.

12. HAPO Study Cooperative Research Group, Metzger BE, Lowe LP, et al. Hyperglycemia and adverse pregnancy outcomes. N Engl J Med. 2008;358(19):1991-2002. https:// doi. org/10.1056/NEJMoa0707943.

13. HAPO Study Cooperative Research Group, Metzger BE, Lowe LP, et al. Hyperglycemia and Adverse Pregnancy Outcome (HAPO) study: associations with neonatal anthropometrics. Diabetes. 2009;58(2):453-9. https://doi.org/10. 2337/db08-1112.

14. Göbl CS, Bozkurt L, Rivic P, et al. A two-step screening algorithm including fasting plasma glucose measurement and a risk estimation model is an accurate strategy for detecting gestational diabetes mellitus. Diabetologia. 2012;55(12):3173-81.https://doi.org/10.1007/s00125012-2726-7.

15. Harreiter J, Kautzky-Willer A. Sex and gender differences in prevention of type 2 diabetes. FrontEndocrinol (Lausanne). 2018;9:220. https://doi.org/10.3389/fendo.2018.00220.

16. Blumer I, Hadar E, Hadden DR, et al. Diabetes and pregnancy: an endocrine society clinical practice guideline. J Clin Endocrinol Metab. 2013;98(11):4227-49. https://doi.org/ $10.1210 /$ jc.2013-2465.

17. Institute of Medicine. Weight gain during pregnancy: reexamining the guidelines. 2009. http://iom.edu/ / media/Files/Report\%20Files/2009/Weight-Gain-DuringPregnancy-Reexamining-the-Guidelines/Report\%20Brief \%20-\%20Weight\%20Gain\%20During\%20Pregnancy.pdf. Zugegriffen:24. Juni2015.

18. Cosson E, Baz B, Gary F, et al. Poor reliability and poor adherence to self-monitoring of blood glucose are common in women with gestational diabetes mellitus and may be associated with poor pregnancy outcomes. Diabetes Care. 2017;40(9):1181-6. https://doi.org/10.2337/dc17-0369.

19. Mendez-Figueroa H, Schuster M, Maggio L, et al. Gestational diabetes mellitus and frequency of blood glucose monitoring: a randomized controlled trial. Obst- et Gynecol. 2017;130(1):163-70. https://doi.org/10.1097/ AOG.0000000000002101.

20. American Diabetes Association. 13. Management of diabetes in pregnancy: standards of medical care in diabetes2018. Diabetes Care. 2018;41(Suppl 1):S137-S43. https:// doi.org/10.2337/dc18-S013.

21. Lv S, Wang J, Xu Y. Safety of insulin analogs during pregnancy: a meta-analysis. Arch Gynecol Obstet. 2015;292(4):749-56. https://doi.org/10.1007/s00404-0153692-3.

22. Milluzzo A, Tumminia A, Scalisi NM, et al. Insulin degludec in the first trimester of pregnancy: report of two cases. J Diabetes Investig. 2017; https://doi.org/10.1111/jdi.12721.

23. Doder Z, Vanechanos D, Oster M, et al. Insulin glulisine in pregnancy - experience from clinical trials and postmarketing surveillance. Eur Endocrinol. 2015;11(1):17-20. https://doi.org/10.17925/EE.2015.11.01.17.

24. Rowan JA, Hague WM, Gao W, et al. Metformin versus insulin for the treatment of gestational diabetes. N Engl J Med. 2008;358(19):2003-15. https://doi.org/10. 1056/NEJMoa0707193.

25. Ijas H, Vaarasmaki M, Morin-Papunen L, et al. Metformin should be considered in the treatment of gestational diabetes: a prospective randomised study. BJOG. 2011;118(7):880-5. https://doi.org/10.1111/j.1471-0528. 2010.02763.x.

26. Balsells M, Garcia-Patterson A, Sola I, et al. Glibenclamide, metformin, and insulin for the treatment of gestational diabetes: a systematic review and meta-analysis. BMJ. 2015;350:h102.https://doi.org/10.1136/bmj.h102.

27. Rowan JA, Rush EC, Obolonkin V, et al. Metformin in gestational diabetes: the offspring follow-up (MiG TOFU): body composition at 2 years of age. Diabetes Care. 2011;34(10):2279-84.https://doi.org/10.2337/dc11-0660.

28. Hanem LGE, Stridsklev S, Juliusson PB, et al. Metformin use in PCOS pregnancies increases the risk of offspring overweight at 4 years of age: follow-up of two RCTs. J Clin Endocrinol Metab. 2018;103(4):1612-21. https://doi.org/ $10.1210 /$ jc. 2017-02419.

29. Camelo Castillo W, Boggess K, Sturmer T, et al. Association of adverse pregnancy outcomes with glyburide vs insulin in women with gestational diabetes. JAMA Pediatr. 2015;169(5):452-8. https://doi.org/10.1001/ jamapediatrics.2015.74.

30. Senat MV, Affres H, Letourneau A, et al. Effect of glyburide vs subcutaneous insulin on perinatal complications among women with gestational diabetes: a randomized clinical trial. JAMA. 2018;319(17):1773-80. https://doi.org/10.1001/ jama.2018.4072.

31. Kautzky-Willer A, Harreiter J. Needle beats pill in gestational diabetes mellitus. Nat Rev Endocrinol. 2018; https://doi. org/10.1038/s41574-018-0050-0.

32. Sutton AL, Mele L, Landon MB, et al. Delivery timing and cesarean delivery risk in women with mild gestational diabetes mellitus. Am J Obstet Gynecol. 2014;211(3):244 e1-244 e7.https://doi.org/10.1016/j.ajog.2014.03.005.

33. Boulvain $\mathrm{M}$, Irion $\mathrm{O}$, Dowswell $\mathrm{T}$, et al. Induction of labour at or near term for suspected fetal macrosomia. Cochrane Database Syst Rev. 2016; https://doi.org/10.1002/14651858. CD000938.pub2.

34. Melamed N, Ray JG, Geary M, et al. Induction of labor before 40 weeks is associated with lower rate of cesarean delivery in women with gestational diabetes mellitus. Am J Obstet Gynecol. 2016;214(3):364 el-364 e8. https://doi. org/10.1016/j.ajog.2015.12.021.

35. Langer O, Berkus MD, Huff RW, et al. Shoulder dystocia: should the fetus weighing greater than or equal to 
$4000 \mathrm{~g}$ be delivered by cesarean section? Am J Obstet Gynecol. 1991;165(4 Pt 1):831-7. https://doi.org/10.1016/00029378(91)90424-P.

36. Gesellschaft für Neonatologie und Pädiatrische Intensivmedizin, Deutsche Gesellschaft für Perinatale Medizin, Deutsche Diabetes Gesellschaft, et al.. Betreuung von Neugeborenen diabetischer Mütter. Frauenarzt. 2003;44(4):439-41.

37. Chertok IR, Raz I, Shoham I, et al. Effects of early breastfeeding on neonatal glucose levels of term infants born to women with gestational diabetes. J Hum Nutr Diet. 2009;22(2):166-9. https://doi.org/10.1111/j.1365-277X. 2008.00921.x.

38. Hillier TA, Pedula KL, Schmidt MM, et al. Childhood obesity and metabolic imprinting: the ongoing effects of maternal hyperglycemia. Diabetes Care.2007;30(9):2287-92.https:/ / doi.org/10.2337/dc06-2361.

39. Ratner RE, Christophi CA, Metzger BE, et al. Prevention of diabetes in women with a history of gestational diabetes: effects of metformin and lifestyle interventions. J Clin Endocrinol Metab. 2008;93(12):4774-9. https://doi.org/10. 1210/jc.2008-0772.

40. Aroda VR, Christophi CA, Edelstein SL, et al. The effect of lifestyle intervention and metformin on preventing or delaying diabetes among women with and without gestational diabetes: the Diabetes Prevention Program outcomes study 10-year follow-up. J Clin Endocrinol Metab. 2015;100(4):1646-53. https://doi.org/10.1210/ jc.2014-3761.

41. Göbl CS, Bozkurt L, Prikoszovich T, et al. Early possible risk factors for overt diabetes after gestational diabetes mellitus. Obstet Gynecol. 2011;118(1):71-8. https://doi.org/10.1097/AOG.0b013e318220e18f.
42. Ziegler AG, Wallner M, Kaiser I, et al. Long-term protective effect of lactation on the development of type 2 diabetes in women with recent gestational diabetes mellitus. Diabetes. 2012;61(12):3167-71. https://doi.org/10.2337/db12-0393.

43. Xiang AH, Kawakubo M, Kjos SL, et al. Long-acting injectable progestin contraception and risk of type 2 diabetes in Latino women with prior gestational diabetes mellitus. Diabetes Care. 2006;29(3):613-7. https://doi.org/10.2337/ diacare.29.03.06.dc05-1940.

44. Shah BR, Retnakaran R, Booth GL. Increased risk of cardiovascular disease in young women following gestational diabetes mellitus. Diabetes Care. 2008;31(8):1668-9. https:// doi.org/10.2337/dc08-0706.

45. Damm P, Houshmand-Oeregaard A, Kelstrup L, et al. Gestational diabetes mellitus and long-term consequences for mother and offspring: a view from Denmark. Diabetologia. 2016;59(7):1396-9. https://doi.org/10.1007/s00125016-3985-5.

46. American Diabetes Association. 2. Classification and diagnosis of diabetes: standards of medical care in diabetes2018. Diabetes Care. 2018;41(Suppl 1):S13-S27.https:// doi. org/10.2337/dc18-S002.

47. Harreiter J, Dovjak G, Kautzky-Willer A. Gestational diabetes mellitus and cardiovascular risk after pregnancy. Womens Health (Lond). 2014;10(1):91-108. https://doi.org/ $10.2217 /$ whe.13.69.

48. World Health Organization. Diagnostic criteria and classification of hyperglycaemia first detected in pregnancy. WHO reference number: WHO/NMH/MND/13.2. 2013. 2013. http://www.who.int/diabetes/publications/ Hyperglycaemia_In_Pregnancy/en/. Zugegriffen: 18. Mai 2015. 